\title{
Colonization of chicks by motility mutants of Campylobacter jejuni demonstrates the importance of flagellin A expression
}

\author{
Trudy M. WassenaAr, ${ }^{1}$ Bernard A. M. van der Zeijst, ${ }^{1 *}$ Roger Ayling ${ }^{2}$ and \\ DiANE G. NEWELL ${ }^{2}$ \\ ${ }^{1}$ Department of Bacteriology, Institute of Infectious Diseases and Immunology, School of Veterinary Medicine, \\ University of Utrecht, PO Box 80.165, 3508 TD Utrecht, The Netherlands \\ ${ }^{2}$ Applied and Molecular Immunology Unit, Central Veterinary Laboratory, New Haw, Weybridge, UK
}

(Received 27 July 1992; revised 10 February 1993; accepted 16 February 1993)

\begin{abstract}
Campylobacter jejuni strain 81116 contains two flagellin genes, $f(a A$ and $f a B$. Wild-type (WT) bacteria express $f a A$ only, but $f a B$ can be expressed under certain conditions. We have determined the importance of flagella for colonization of the avian caecum, which appears to be the natural environment for these bacteria. Mutants in which $f a A$ or $f a B$, or both had been inactivated, and motility variants, were investigated. Flagella are not a requisite for colonization, but mutants lacking both flagellin genes colonized less efficiently than WT. Inactivation of the $f a B$ gene, which had no effect on bacterial motility, enhanced chicken caecal colonization 1000-fold compared to WT. A variant (SF-1) with flagella composed of flagellin A, but with poor motility, also colonized better than WT. Conversely, mutants with an inactivated $f a A$ gene colonized 100 - to 1000 -fold less efficiently than WT, regardless of their motility conferred by truncated or full-length flagellin B flagella. These results suggest that the presence of flagellin A, rather than motility, is essential for optimal bacterial colonization of chicken caeca.
\end{abstract}

\section{Introduction}

Campylobacter jejuni infection is a major cause of diarrhoea in humans (Blaser \& Reller, 1981). In contrast, the organism is a commensal of poultry, from which it enters the human food chain (Harris et al., 1986; Stern et al., 1988; Shane, 1992). In order to reduce the prevalence of $C$. jejuni in chickens, and thus decrease the risk of human infection, a better understanding of the factors involved in avian colonization is needed. Successful colonization involves a complex interaction between the host and bacterium. For Campylobacter colonization in the chicken, the factors involved in such interactions can only be speculative. However, a chicken colonization model has recently been developed (Stern et al., 1988), which may allow bacterial colonization factors to be identified (Meinersmann, 1990).

The pathogenesis of campylobacteriosis is also largely unknown, but several virulence factors have been identified including cytotoxins (Guerrant et al., 1987; Mahajan \& Rodgers, 1990), adhesins (De Melo \& Pechère, 1990) and flagella (Newell et al., 1985; Morooka et al., 1985). The flagella, which confer motility upon the

* Author for correspondence. Tel. 30 534888; fax 30540784.

Abbreviations: WT, wild-type; CC, caecal contents. bacteria, are, to date, the most investigated virulence factor. In vitro studies have established a role for flagella in invasion (Wassenaar et al., 1991), possibly via adherence (Newell et al., 1985). Furthermore, the importance of motility in colonization has been demonstrated in several animal models, including mice (Morooka et al., 1985; Newell et al., 1985), rabbits (Caldwell et al., 1985; Pavlovskis et al., 1991) and hamsters (Aguero-Rosenfeld et al., 1990). Volunteer experiments also suggest a role for flagella in human infection (Black et al., 1988), but as yet the role in chicken colonization has not been investigated.

Both $C$. jejuni and C. coli, possess two flagellin genes, flaA and flaB (Nuijten et al., 1990; Guerry et al., 1990). In $C$. coli strain VC167, both flagellin genes are expressed and their products are incorporated together into flagella, with flagellin A as the major component (Guerry et al., 1990, 1991). In contrast, C. jejuni strain 81116 is able to produce two types of flagella, depending on which flagellin gene is expressed. In the wild-type (WT) bacteria of this strain, only $f a A$ expression is detectable (Nuijten et al., 1990). The flaB gene of this strain can be inactivated without loss of motility or invasiveness, as determined in vitro (Wassenaar et al., 1991). In contrast, the inactivation of flaA leads to a 100 -fold decrease in these properties. This suggests that the two types of 
Table 1. A comparison of motility, invasive properties in vitro and colonization in vivo of the C. jejuni 81116 strains used in this study.

Motility was determined on motility media. The genotype was determined by Southern blotting (Wassenaar et al., 1991). The nature of the flagellin was determined on Western blots using monoclonal antibody specific for flagellin A (Nuijten et al., 1991) and polyclonal antibody specific for flagellins A and B (Nuijten et al., 1989; Wassenaar et al., 1991). The average level of colonization of chicks dosed with approximately $5 \times 10^{3}$ or $10^{5}$ c.f.u. is given. ND, Not determined.

\begin{tabular}{lccccc}
\hline \hline & & & & \multicolumn{2}{c}{ In vivo colonization: } \\
\cline { 5 - 6 } Strain & Motility & Genotype & $\begin{array}{c}\text { Composition } \\
\text { of flagella }\end{array}$ & dosed at $5 \times 10^{3}$ & dosed at $10^{5}$ \\
\hline WT & +++ & $\mathrm{A}^{+} \mathbf{B}^{+}$ & FlaA $^{*}$ & $10^{6}$ & $10^{9}$ \\
R1 & + & $\mathrm{A}^{-} \mathbf{B}^{+}$ & FlaB & ND & $10^{6}$ \\
R1-V2 & ++ & $\mathrm{A}^{-} \mathbf{B}^{+}$ & FlaB & ND & $10^{4}$ \\
R2 & - & $\mathrm{A}^{-} \mathbf{B}^{-}$ & None & ND & $10^{7}$ \\
R3 & +++ & $\mathrm{A}^{+} \mathbf{B}^{-}$ & FlaA & $10^{9}$ & ND \\
SF-1 & ++ & $\mathrm{A}^{+} \mathbf{B}^{+}$ & FlaA* & $10^{9}$ & ND \\
\hline \hline
\end{tabular}

${ }^{*}$ No flagellin B was detectable, but the presence of minor amounts cannot be excluded.

$\dagger R 1$ produces truncated flagella, consisting of flagellin B.

flagella produced by $C$. jejuni strain 81116 have markedly different biological properties. The importance of these two types of flagella for survival in a natural environment is still unknown. To establish their role in colonization of the avian caecum, isogenic mutants expressing $\mathrm{flaA}$, $f a B$ or neither gene were investigated in the chick model.

\section{Methods}

Bacterial strains. C. jejuni 81116 has been extensively passaged in vitro since its isolation from a patient suffering from diarrhoea (Palmer et al., 1983). Fully motile colonies from thioglycollate plates containing $0.4 \%$ agar (motility media) (Caldwell et al., 1985), were cloned to purity and designated as wild-type (WT). Flagellin mutants R1, R2 and R3 were produced by homologous recombination with a vector containing a kanamycin-resistance gene flanked by flagellin sequences (Wassenaar et al., 1991). Mutant R1 has an inactivated flaA gene and flagellin $B$ is assembled into truncated flagella. Mutant $R 2$ is a deletion mutant lacking part of $f a A$ and $f a B$, and, therefore, has no remaining functional flagellin gene. Mutant R3 lacks a functional $f a B$ gene and is completely motile by means of flagella consisting of flagellin A. Mutant R1-V2 is a spontaneous variant of R1 that has partly regained motility, due to a transcriptional upshift of $f a B$ (T. M. Wassenaar and others, unpublished). SF-1 is a spontaneous mutant of WT that was originally described as a non-motile mutant, since it forms pin-point colonies in motility plates containing $0.7 \%$ agar (Newell et al., 1984). However, in thioglycollate plates containing $0.4 \%$ agar this variant has a motility comparable to that of R1-V2. The properties of the WT strain and all mutants of $C$. jejuni 81116 used in this study are summarized in Table 1.

Culture conditions. All strains were cultured on Skirrow's selective media (Skirrow, 1977) with $10 \%(\mathrm{v} / \mathrm{v})$ sheep blood at $42{ }^{\circ} \mathrm{C}$ in an atmosphere of $7 \% \mathrm{CO}_{2} / 8 \% \mathrm{O}_{2} / 85 \% \mathrm{~N}_{2}$. For kanamycin selection, plates were supplemented with $30 \mu \mathrm{g}$ kanamycin $\mathrm{ml}^{-1}$. For Campylobacter selection from chicken caecal contents $30 \mu \mathrm{g}$ cephaperazone $\mathrm{ml}^{-1}$ was added to suppress normal caecal flora. In each experiment, bacterial motility was monitored prior to inoculation and after colonization by colony size in motility media.
Infection of chickens. Chicks were colonized as previously described by Stern et al. (1988). Eggs from a Torbay 9 flock (Wickham Laboratories, UK) were hatched in isolators. Groups of six to ten birds were maintained in isolators and provided with unlimited food and water. Campylobacter strains were cultured overnight, harvested in phosphate-buffered saline (PBS; $6.5 \mathrm{~mm}-\mathrm{Na}_{2} \mathrm{HPO}_{4}, 1.5 \mathrm{~mm}-\mathrm{KH}_{2} \mathrm{PO}_{4}$, $137 \mathrm{~mm}-\mathrm{NaCl}, 1.5 \mathrm{~mm}-\mathrm{KCl}, \mathrm{pH} 7 \cdot 3$ ), and washed and diluted in PBS. Doses were estimated by measuring the $A_{280}$ of an aliquot of lysed cells and determined against a standard curve. The actual doses were measured by performing viable counts on blood agar plates. Approximately $24 \mathrm{~h}$ after hatching, chicks were dosed by gavage with $0.1 \mathrm{ml}$ bacterial suspensions or $0.1 \mathrm{ml}$ PBS; $5 \mathrm{~d}$ after this inoculation, the birds were sacrificed by cervical dislocation. The chicks were weighed, the abdomen skinned and the abdominal contents gently removed to observe gross pathology. The contents $(\mathrm{CC})$ of one caecum were gently extruded, weighed and diluted to give $0.1 \mathrm{~g} \mathrm{CC} \mathrm{ml}^{-1}$ in PBS. The weight of the caecal contents varied from $0 \cdot 1$ to $0 \cdot 7 \mathrm{~g}$. Sequential dilutions were plated out on selective blood agar plates, with and without kanamycin, and cultured for $48 \mathrm{~h}$ to give viable colony counts. In addition, dilutions were cultured in motility medium. The native micro-aerophilic faecal flora were quantified by performing viability counts on blood agar plates without antibiotics.

\section{Results}

The colonization dose response (of between 54 and $5 \times 10^{5}$ bacteria) in 1-d-old chicks was determined for C. jejuni 81116 WT (Fig. 1). Six groups of chicks were infected with various doses of bacteria and the level of colonization was determined after $5 \mathrm{~d}$. No Campylobacter colonization was detectable in any of the control birds dosed with PBS. An infective dose of 54 colonyforming units (c.f.u.) Campylobacter per chick colonized three out of eight animals. The detection level of colonization was $10^{2}$ c.f.u. $(\mathrm{g} \mathrm{CC})^{-1}$. As expected, there was a direct relationship between infective dose and colonization levels. All chicks dosed with $5 \times 10^{3}$ c.f.u. or more were infected. Neither weight loss nor gross 


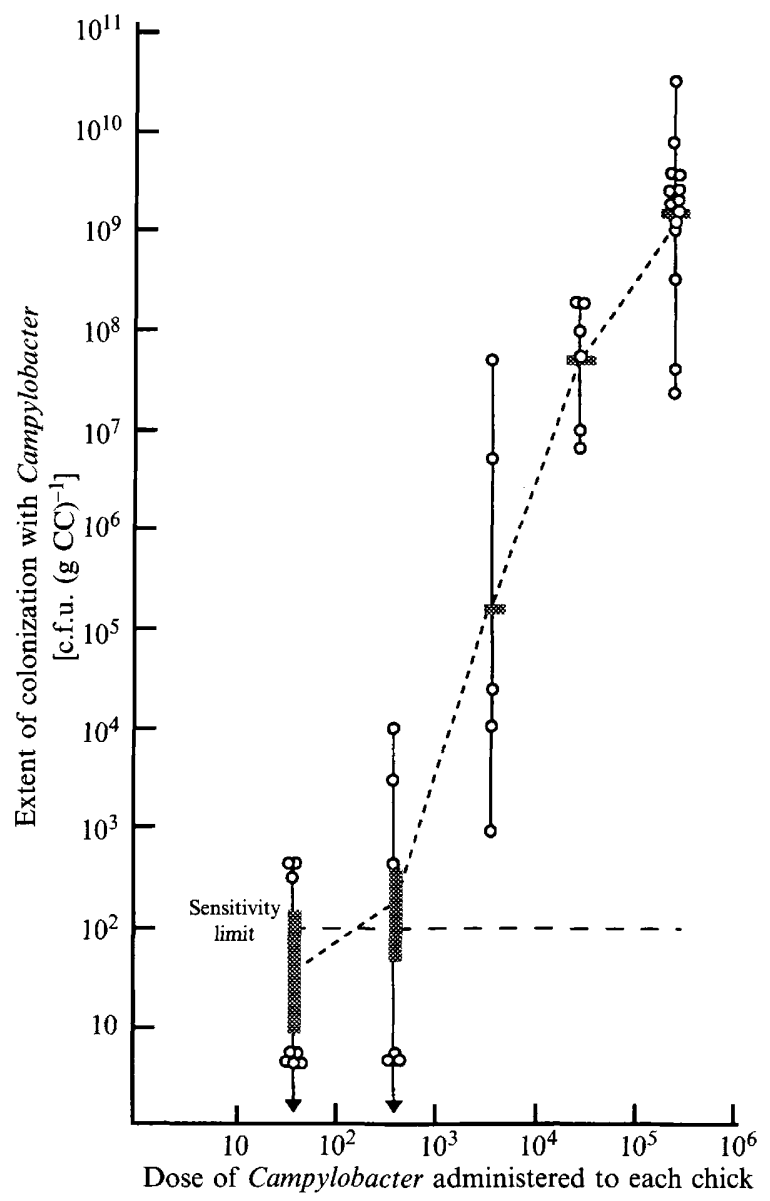

(c.f.u.)

Fig. 1. Caecal colonization of chicks orally infected with various numbers of $C$. jejuni WT. Colonization of each chick with Campylobacter $(\mathrm{O})$ is given per g CC $6 \mathrm{~d}$ post-infection. The geometric mean of the colonization levels of each group (1) and mean infective dose response curve (-----) are given. In the first two groups a further five and three chicks, respectively, had fewer than $10^{2}$ c.f.u. $(\mathrm{g} \mathrm{CC})^{-1}$, which is the sensitivity limit. The means of these groups were therefore calculated with the maximum variation of 0 to 99 c.f.u. $(\mathrm{g} \mathrm{CC})^{-1}$ for these chicks.

pathological changes were observed in the colonized chicks.

The presence of Campylobacter did not lead to a decrease in the native micro-aerophilic microflora of the caecum, as determined by colony counts on plates without selective antibiotics. In the highest dosed groups, Campylobacter outnumbered the native flora by a factor of 100 .

Chicks were inoculated with the motility mutants. The target dose for R1 and R2 was $10^{5}$ c.f.u. per chick, which was above the $100 \%$ colonization dose for WT because these mutants were expected to colonize poorly. Mutant R3, which was expected to colonize as efficiently as WT, and variant SF1, which had been shown to colonize as well as WT in the infant mouse model (Newell et al.,

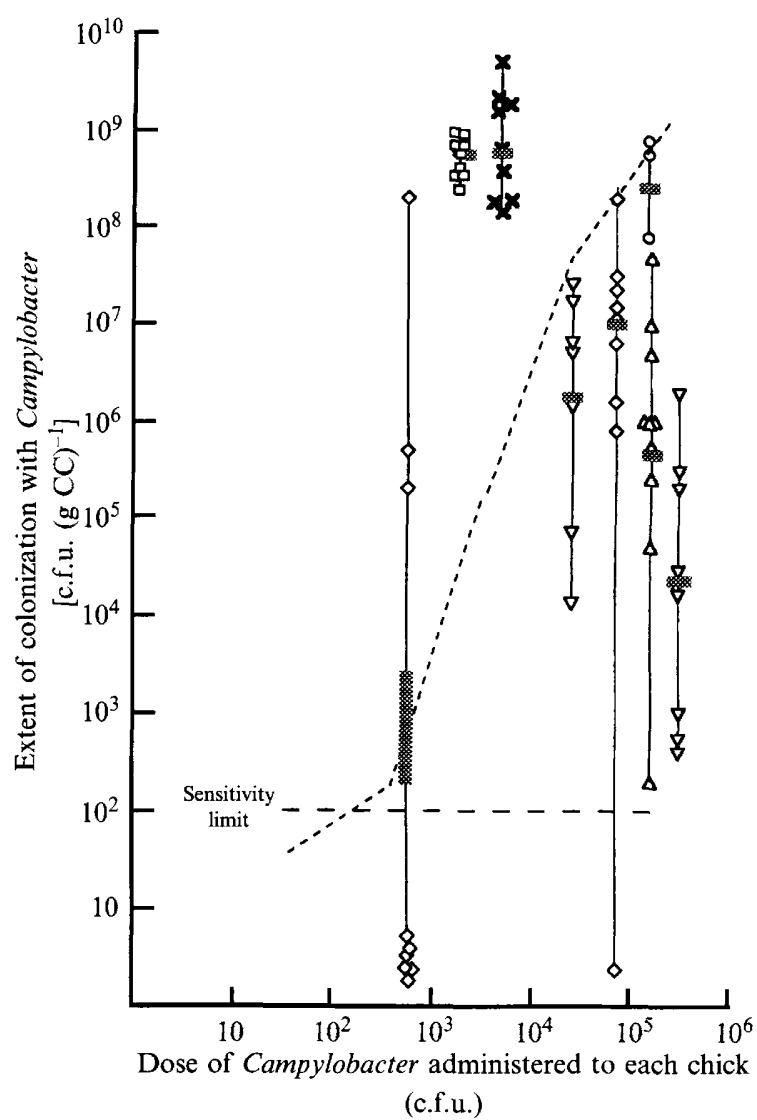

Fig. 2. Caecal colonization of chicks orally infected with WT $(O)$, SF-1 $(\square)$, R3 $(\times)$, R2 $(\diamond), \mathrm{R} 1(\triangle)$ and R1-V2 $\nabla)$. Chicks were infected with R2 and R1-V2 at two different doses. Colonization of each chick with Campylobacter is given per g CC $6 \mathrm{~d}$ post-infection. The geometric

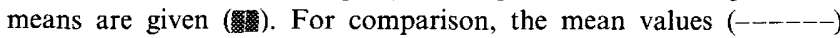
obtained with various doses of WT, as determined in Fig. 1, is given.

1985), had target doses of $10^{3}$ c.f.u. per chick. Mutant R1-V2 was tested at two doses $\left(4 \times 10^{4}\right.$ and $2 \times 10^{5}$ c.f.u. per chick) because there was no information available about its colonization potential. For each experiment, the WT strain was included as a positive control at a dose of approximately $10^{5}$ c.f.u. per chick. In each experiment the colonization potential of WT was reproducible. The ability of the mutants to colonize the chicks varied significantly (Fig. 2). Mutant R2, lacking both flagellin genes (Table 1), colonized only slightly less efficiently than WT. To determine whether this relatively high colonization level was due to the high dose, R2 was also tested at the lower dose of $10^{3}$ c.f.u. per chick. This resulted in a mean colonization level equal to that of WT, but only three out of nine birds were colonized. Mutant R3, lacking a $f a B$ gene, colonized chicks 1000 times more efficiently than WT at a dose of $5 \times 10^{3}$ c.f.u. per chick. Conversely, mutant $\mathrm{R} 1$, which produces truncated flagellin B flagella (Table 1), colonized chicks 1000 times less efficiently than WT. Similarly, the motility variant of $\mathrm{R} 1$ (R1-V2) colonized to about the same extent as R1 
(between 10 - and $10^{4}$-fold less than WT), depending on the dose. Unlike WT, colonization by mutant R1-V2 apparently reached a maximum at a dose of $3.4 \times 10^{4}$ c.f.u. per chick, since a dose of $2 \times 10^{5}$ c.f.u. per chick did not further increase colonization levels. Interestingly SF-1, which has a motility comparable to R1-V2, was able to colonize the chicken caeca much better, to the same extent as R3. Mutants R1 and R2 had a wider range of colonization than the other mutants or WT. The reasons for this are unknown.

All bacterial strains recovered from the caeca displayed the original colony morphology on motility media, indicating that there was no in-vivo-induced or selected change in motility. Variant R1-V2 can revert to the R1 phenotype at a low frequency in vitro (T. M. Wassenaar and others, unpublished). Nevertheless, R1-V2 and R1 recovered from caecal contents of chicks retained their original phenotype, so a switch in flagellin phenotype from $\mathrm{R} 1$ to $\mathrm{R} 1-\mathrm{V} 2$ or vice versa does not appear to be inducible by colonization.

\section{Discussion}

C. jejuni appears to be non-pathogenic in chickens, even though chicken caeca can be highly colonized (Stern et al., 1988; Shane, 1992). The bacterial factors involved in the colonization of the avian caeca are unknown. However, it seemed likely that effective motility is a prerequisite of survival in such a mucoid microenvironment (Lee et al., 1986). Motility in Campylobacter is mediated by the products of two flagellin genes, $f a A$ and $f a B$. In this study, we made use of isogenic mutants of $C$. jejuni 81116 to differentiate between the relative importance for caecal colonization of motility and the mere presence of the two types of flagellin. Our results clearly demonstrate that flagellin expression and motility are not essential for colonization, although the nature of the flagellin expressed has a significant effect on the ability of the bacterium to colonize chicken caeca.

The lack of correlation between bacterial motility and avian caecal colonization (Table 1 ) is exemplified by the difference in motility between $\mathrm{R} 1$ and $\mathrm{R} 1-\mathrm{V} 2$, which is not reflected in their colonization capacity. Moreover, SF-1, displaying a motility similar to R1-V2, colonizes 1000 -fold better than WT, and the completely nonmotile mutant R2 colonizes as efficiently as R1. The finding that motility is not required for colonization is in contrast to data from previous animal model studies (Newell et al., 1985; Morooka et al., 1985; Pavlovskis et al., 1991). Possible explanations for this anomaly are the differences in the animal models and the motility mutants used. Morooka et al. (1985) and Newell et al. (1985) used non-defined motility mutants and variants to demonstrate the importance of motility for colonization in a suckling mouse model. Such mutants may have had concomitant changes in other colonization factors. An isogenic aflagellate mutant was unable to colonize rabbits in either an oral or a RITARD model (Pavlovskis et al., 1991). However, the artificial nature of the RITARD model, competition with pre-existing gut flora, and host and site differences in mucous composition, are likely reasons for the discrepancy.

The chicken colonization model is a reflection of the micro-environment that $C$. jejuni naturally encounters. The results obtained in this study indicate the relative unimportance of bacterial motility for survival in such a unique niche.

Although motility is not a prerequisite for colonization, the nature of the flagellin still has an effect on colonization potential. Mutants expressing flagellin B (R1 and R1-V2) display colonization levels that are at least 100-fold lower than WT, SF1 and R3, all expressing flagellin A.

The expression of flagellin B by $C$. jejuni is apparently not advantageous to caecal colonization. Flagellin mutant R3, lacking the $f a B$ gene, colonizes much more efficiently than WT. This was unexpected since $f a B$ expression was undetectable in W1 (Nuijten et al., 1990). Nevertheless, this anomaly could be explained by a low expression level of $f l a B$ in WT, which in some undefined way affects colonization potential. Another, more likely, explanation is that inactivation of $f a B$ may influence the activity of other, as yet unidentified, colonization factors.

The mutants expressing flagellin B used in this study contain an inactivated $f a A$ gene. However, we have observed that culturing wild-type $C$. jejuni 81116 in vitro can result in a switch to flagellin B expression (T. M. Wassenaar and others, unpublished). It can therefore be hypothesized that bacteria surviving an in vitro environment partly express flagellin $\mathrm{B}$. The colonization potential of $\mathrm{R} 1$ and $\mathrm{R} 1-\mathrm{V} 2$ suggests that such bacteria are less able to colonize chickens than bacteria expressing flagellin A.

The importance of factors other than flagella in the colonization of chicken caeca remains to be investigated. Some Campylobacter strains undergo antigenic changes during chicken colonization (Meinersmann et al., 1990), indicating that novel bacterial components are expressed during colonization. The occurrence of such antigenic changes, which could be due to the selection of preexisting mutants or the induction of genes coding for these components, is currently under investigation.

\footnotetext{
We thank S. Cawthraw for help with the laborious work of plating out caecal content dilutions. This work was supported by the Netherlands Foundation for Chemical Research (SON) with financial aid from the Netherlands Organization for Scientific Research (NWO), and by the Ministry of Agriculture, Food and Fisheries, UK.
} 


\section{References}

Aguero-Rosenfeld, M. E., YANG, X. H. \& Nachamkin, I. (1990). Infection of Syrian hamsters with flagellar variants of Campylobacter jejuni. Infection and Immunity 58, 2214-2219.

Black, R. E., Levine, M. M., Clements, M. L., Hughes, T. P. \& BlASER, M. J. (1988). Experimental Campylobacter jejuni infection in humans. Journal of Infectious Diseases 157, 472-479.

Blaser, J. M. \& Reller, L. B. (1981). Campylobacter enteritis. New England Journal of Medicine 305, 1444-1452.

Caldwell, M. B., Guerry, P., Lee, E. C., Burans, J. P. \& Walker, R. I. (1985). Reversible expression of flagella in Campylobacter jejuni. Infection and Immunity 50, 941-943.

De Melo, M. A. \& Pechìre, J.-C. (1990). Identification of Campylobacter jejuni surface proteins that bind to eucaryotic cells in vitro. Infection and Immunity 58, 1749-1756.

Guerrant, R. L., Wanke, C. A., Pennie, R. A., Barrett, L. J., Lima, A. A. M. \& O'Brien, A. D. (1987). Production of a unique cytotoxin by Campylobacter jejuni. Infection and Immunity 55, 2526-2530.

Guerry, P., Logan, S. M., Thornton, S. \& Trust, T. J. (1990). Genomic organization and expression of Campylobacter flagellin genes. Journal of Bacteriology 172, 1853-1860.

Guerry, P., Alm, R. A., Power, M. E., Logan, S. M. \& Trust, T. J. (1991). Role of two flagellin genes in Campylobacter motility. Journal of Bacteriology 173, 4757-4764.

Harris, N. V., Weiss, N. S. \& Nolan, C. M. (1986). The role of poultry and meats in the etiology of Campylobacter jejuni/coli enteritis. American Journal of Public Health 76, 407-411.

MAHAJAN, S. \& RoDGERS, F. G. (1990). Isolation, characterization and host-cell-binding properties of a cytotoxin from Campylobacter jejuni. Journal of Clinical Microbiology 28, 1314-1320.

Meinersmann, R. J., Stern, N. J. \& Blankenship, L. C. (1990). Antigenic differences in congenic chicken-colonizing and noncolonizing strains of Campylobacter jejuni. Current Microbiology 21, $17-21$.

Morooka, T., UmeKa, A. \& Amako, K. (1985). Motility as an intestinal colonization factor for Campylobacter jejuni. Journal of General Microbiology 131, 1973-1980.
Lee, A., O'Rourke, J., Barrington, P. J. \& Trust, T. J. (1986) Mucus colonization as a determinant of pathogenicity in intestinal infection by Campylobacter jejuni: a mouse caecal model. Infection and Immunity 51, 5536-546.

Newell, D. G., McBride, H. \& Pearson, A. D. (1984). The identification of outer membrane proteins and flagella of Campylobacter jejuni. Journal of General Microbiology 130, 1201-1208.

NewELl, D. G., MCBRIDE, G. \& DolBY, J. M. (1985). Investigations on the role of flagella in the colonization of infant mice with Campylobacter jejuni and attachment of Campylobacter jejuni to human epithelial cell lines. Journal of Hygiene 95, 217-227.

Nuijten, P. J. M., Bleumink-Pluym, N. M. C., GaAstra, W. \& van DER ZEIJST, B. A. M. (1989). Flagellin expression in Campylobacter jejuni is regulated at the transcriptional level. Infection and Immunity 57, $1084-1088$.

Nuijten, P. J. M., van Asten, F. J. A. M., Gaastra, W. \& van der ZeiJst, B. A. M. (1990). Structural and functional analysis of two Campylobacter jejuni flagellin genes. Journal of Biological Chemistry 265, 17798-17804.

Nuijten, P. J. M., Van der Zeisst, B. A. M. \& Newell, D. G. (1991). Localization of immunogenic regions on the flagellin proteins of Campylobacter jejuni 81116. Infection and Immunity 59, 1100-1105.

Palmer, S. R., Gully, P. R., White, J. M., Pearson, A. D., Suckling, W. G., Jones, D. M., Rawes, J. C. L. \& Penner, J. L. (1983). Waterborne outbreak of Campylobacter gastroenteritis. Lancet $i, 287-290$.

Pavlovskis, O. R., Rollins, D. M., Haberberger, R. L., Green, A. E., Habash, L., Strocko, S. \& Walker, R. I. (1991). Significance of flagella in colonization resistance of rabbits immunized with Campylobacter spp. Infection and Immunity 59, 2259-2264.

SHANE, S. M. (1992). The significance of Campylobacter jejuni infection in poultry: a review. Avian Pathology 21, 189-213.

SkIRRows, M. B. (1977). Campylobacter enteritis - a new disease. British Medical Journal 2, 9-11.

Stern, N. J., Bailey, J. S., Blankenship, L. C., Cox, N. A. \& McHan, F. (1988). Colonization characteristics of Campylobacter jejuni in chick caeca. Avian Diseases 32, 330-334.

Wassenaar, T. M., Bleumink-Pluym, N. M. C. \& VAN der Zeist, B. A. M. (1991). Inactivation of Campylobacter jejuni flagellin genes by homologous recombination demonstrates that $f a A$ but not $f l a B$ is required for invasion. EMBO Journal 10, 2055-2061. 